\title{
Anaplastic Large Cell Lymphoma
}

National Cancer Institute

\section{Source}

National Cancer Institute. Anaplastic Large Cell Lymphoma. NCI Thesaurus. Code C3720.

A peripheral (mature) T-cell lymphoma, consisting of usually large anaplastic, CD30 positive cells. The majority of cases are positive for the anaplastic large cell lymphoma (ALK) protein. The most frequently seen genetic alteration is a $t(2 ; 5)$ translocation. Majority of patients present with advanced disease. The most important prognostic indicator is ALK positivity, which has been associated with a favorable prognosis. (WHO, 2001) 